\title{
Hippocampal Long-Lasting Potentiation Produced by Pairing Single Volleys and Brief Conditioning Tetani Evoked in Separate Afferents
}

\author{
B. Gustafsson and H. Wigström \\ Department of Physiology, University of Göteborg, S-400 33, Göteborg, Sweden
}

Cooperative effects between afferents on the induction of longlasting potentiation (LLP) of synaptic transmission were examined in the CA1 region of the hippocampal slice preparation. Synaptic activity was recorded extracellularly in the dendritic layer (stratum radiatum) as a field EPSP, and the amount of LLP produced was measured from the change in slope of the rising phase of this potential. Experiments were performed with the GABA antagonist picrotoxin in the bath solution in order to facilitate the induction of LLP. It is shown that under these conditions a test input evoked by single volleys (at $0.2 \mathrm{~Hz}$ ) is potentiated when paired with brief tetani (2-15 impulses at 50 Hz) to a separate population of fibers in the stratum radiatum. For this potentiation to appear, the test input had to occur durIng the train or precede it by less than $\mathbf{4 0}$ msec. Maximal effects were observed with the test volley positioned in the early part of the tetanus, and were largely independent of train duration. The potentiation obtained in this manner reached a peak level after some 20 conjunction events, and its magnitude measured 10 min after conjunction was about half that which could be induced by homosynaptic tetanization. Prior homosynaptic potentiation occluded the potentiation induced by conjunction, suggesting an identity of their underlying mechanisms. A test input to the apical dendritic layer was potentiated also by inputs to the basal dendritic layer, although greater effects were observed with both inputs in the same dendritic layer. It is suggested that the conditioning effect is related to the postsynaptic depolarization created by the tetanus. The results support the recent proposal that LLP is induced by ionic flux through voltage-dependent synaptic channels.

In the hippocampus, tetanic activation of afferents leads to a very prolonged increase in synaptic efficacy, known as longlasting potentiation (LLP) (Bliss and Gardner-Medwin, 1973; Bliss and Lømo, 1973; Douglas and Goddard, 1975; Lømo, 1966). Even though considerable work has been devoted to finding the mechanism(s) underlying LLP, this question is still unsettled, and there is even controversy whether the actual modification takes place on the pre- or postsynaptic side (e.g., see Dolphin et al., 1982; Douglas et al., 1982; Lynch et al., 1983; Skrede and Malthe-Sørenssen, 1981; see also Bliss and Dolphin, 1982).

Under normal conditions, LLP is induced by long stimulus trains giving rise to considerable activity in both presynaptic terminals and postsynaptic cells. It has recently been shown, however, that the induction of LLP is greatly facilitated when

\footnotetext{
Received Apr. 2, 1985; revised Oct. 28, 1985; accepted Oct. 30, 1985.

We thank Dr. W. C. Abraham for helpful discussions. This work was supported by the Swedish Medical Research Council (Projects 05954 and 05180), the Swedish Natural Science Research Council (Project 4018), and Magnus Bergvalls Stiftelse.

Correspondence should be addressed to Dr. Gustafsson, Department of Physiology, University of Göteborg, Box 33031, S.400 33 Göteborg, Sweden.

Copyright (C) 1986 Society for Neuroscience $0270-6474 / 86 / 061575-08 \$ 02.00 / 0$
}

postsynaptic inhibition is reduced by application of GABA antagonists (Wigström and Gustafsson, 1983a, b, 1985b); a substantial potentiation could then appear after only a few afferent volleys. It would seem that analysis of LLP in such a preparation would help to clarify questions regarding the relative importance that the different processes involved in synaptic transmission may have for the induction of LLP, and thereby its likely site of generation.

The results observed following application of GABA antagonists were interpreted as suggesting that the generation of LLP was controlled to a significant extent by postsynaptic activity, the facilitated induction of LLP being due to the greater degree of postsynaptic activity obtained during a tetanus when inhibition is reduced. The low number of afferent volleys needed to evoke LLP may then imply that tetanic activation of the synaptic terminals themselves is not a requirement for the generation of LLP, except as a means to produce the necessary postsynaptic activity. If this is true, the response even to a single afferent volley might become potentiated if it occurs in conjunction with the postsynaptic activity elicited by a brief tetanus to another input. It will be shown that in the presence of picrotoxin an LLP can be induced under such conditions if the temporal relations between the test volley and the conditioning tetanus are appropriate. The results support the view that the generation of LLP is controlled by processes occurring within the postsynaptic neuron (Douglas et al., 1982; Eccles, 1983; Lynch and Baudry, 1984; Lynch et al., 1983; Wigström and Gustafsson, 1985b).

\section{Materials and Methods}

Experiments were performed on hippocampal slices from 20 guinea pigs using standard techniques (Skrede and Westgaard, 1971). The animals were anesthetized with ether and their brains were removed. The hippocampus on one side was dissected out and cut into $400 \mu \mathrm{m}$ thick transverse slices. During the experiment, the slices were kept on a Nylon net in a constant-flow incubation chamber at $30-32^{\circ} \mathrm{C}$, half-submerged by a solution containing (in $\mathrm{mM}$ ): $\mathrm{NaCl}, 124 ; \mathrm{KCl}, 4 ; \mathrm{CaCl}_{2}, 4 ; \mathrm{MgCl}_{2}$, $4 ; \mathrm{NaHCO}_{3}, 26$; glucose, 10; picrotoxin, 0.1 . Concentrations of calcium and magnesium in this solution are somewhat higher than normal in order to counteract the excitability increase obtained in the presence of picrotoxin. Thus, spontaneous repetitive field potentials or late discharges following stimulation were not observed in these slices (Hablitz, 1984). Normally, picrotoxin was added about $2 \mathrm{hr}$ after the incubation of the slices.

In the present investigation, subfield CA1 was used (see Fig. 1A). Electrical stimulation of afferent fibers and recording of extracellular synaptic field potentials were carried out in the apical dendritic layer (stratum radiatum) of the $\mathrm{C} \Lambda 1$ pyramidal cells (Fig. 1B). Stimuli consisted of $0.1 \mathrm{msec}$ negative constant-current pulses $(10-30 \mu \mathrm{A})$ delivered through electrolytically sharpened tungsten wires (monopolar stimulation). For tetanic activation, short (2-15 impulses) $50 \mathrm{~Hz}$ trains were used. When two independent inputs were needed, the stimulus electrodes were generally positioned on either side of the recording electrode (STIM 1 and SIIM 2, Fig. $1 B$ ). The independence of the two inputs 

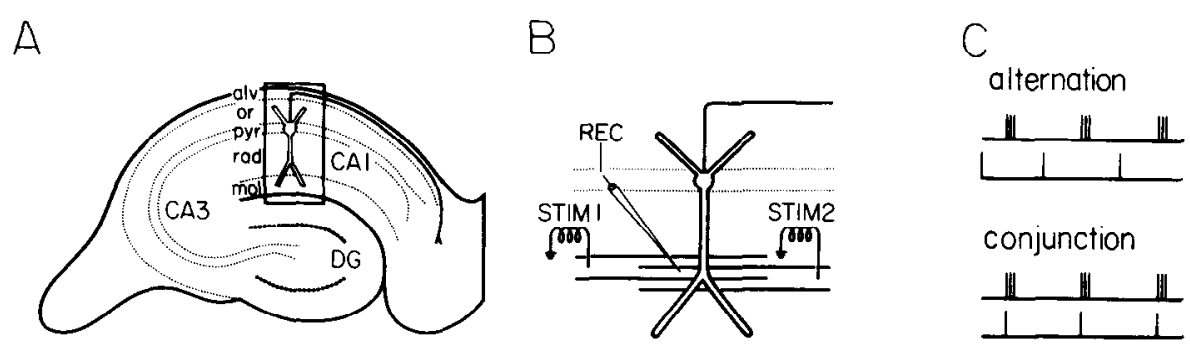

D
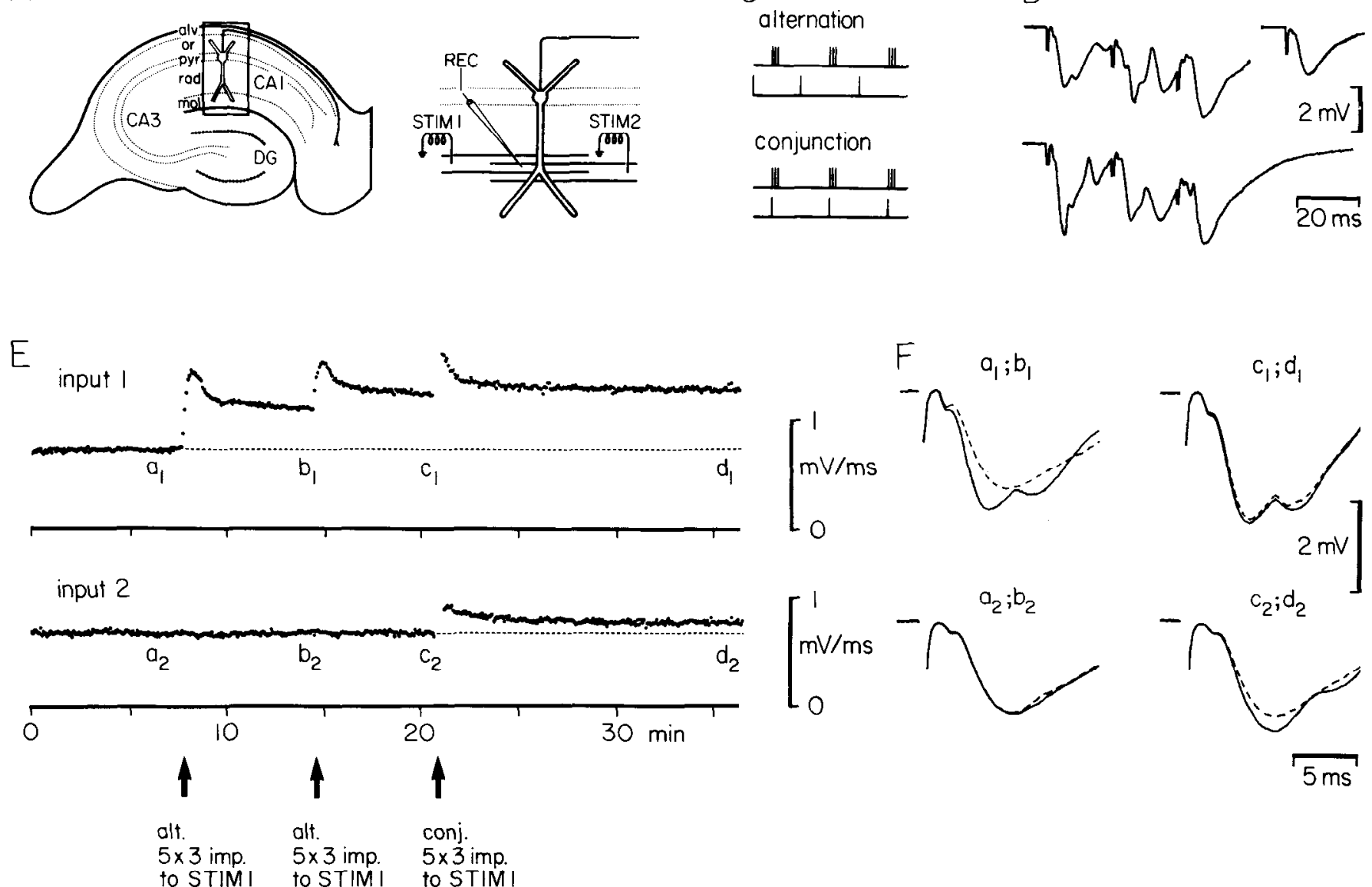

Figure 1. LLP induced by combining single volleys and brief tetani to separate afferents. $A$, Schematic drawing of the hippocampal slice with the studied CA 1 region boxed in. Abbreviations: alv, alveus; or, stratum oriens; $p y r$, stratum pyramidale; rad, stratum radiatum; mol, stratum moleculare. $B$, Diagram showing the arrangement of stimulating $(S T I M)$ and recording $(R E C)$ electrodes. $C$, Schematic drawing of the stimulus conditions during tetanization. In the alternation mode (upper half), the stimuli to STIM 1 and STIM 2 were kept 2.5 sec apart, while in the conjunction mode (lower half), the stimulus to STIM 2 coincided with the first stimulus to STIM $1 . D$. Sample records of extracellular responses from the apical dendritic layer, stratum radiatum. Upper records show the responses to a three-impulse tetanus to STIM 1 and a single stimulus to STIM 2, while the lower record shows the response to a combined activation of STIM 1 and STIM 2. E, Measurements of the initial slopes of the field EPSPS resulting from activation of STIM 1 (input 1 ) and STIM 2 (input 2) are shown for a series of test responses. Input 1 was subjected to a series of tetanic activations as indicated in the bottom of the graph. $F$, Average records $(n=10)$ of the responses shown in $E$, taken at the indicated times. Upper records are responses to STIM 1 (input 1 ) and lower records to STIM 2 (input 2).

was ascertained by a volley summation test, i.e., that the algebraical sum of the presynaptic volleys in response to STIM 1 and STIM 2 was equal to the response given by a simultaneous activation of the two inputs (see Wigström and Gustafsson, 1985b). In some experiments in which three stimulus electrodes were used (see Fig. $2 A$ ), the independence of the inputs was tested in a similar way. Population spikes measured in the cell body layer were generally larger following simultaneous activation of two separate inputs than following stimulation with double strength of a single input (Wigström and Gustafsson, 1985b). This indicates a large degree of convergence of the separate inputs onto the same postsynaptic neurons.

Recordings were made in the stratum radiatum layer by means of glass micropipettes filled with $3 \mathrm{M} \mathrm{NaCl}$ (5-15 M $\Omega$ resistance). Examples of recorded potentials are shown in Figure $1 F$. After passing through a high-impedance preamplifier, the signals were connected to an analogto-digital converter and fed into a NORD-100 minicomputer. The latter was used for on-line signal averaging and for measurement of waveform parameters. The presynaptic volley was calculated as the peak-to-peak amplitude of the initial small deflection, while the field EPSP was taken as the initial slope of the following negative wave. The computed waveform parameters as well as digital records of original single or averaged potentials were stored in the mass memory of the computer for later off-line analysis. Hard copies of experimental data were generated by a pen plotter.

\section{Results}

As shown in Figure $1 E$ (third arrow), in the presence of picrotoxin in the bath, a single volley test EPSP (input 2 ) increased in magnitude when paired a few times at low frequency $(0.2$ $\mathrm{Hz}$ ) with a brief tetanus (three impulses) to separate afferents (input one) projecting to the same dendritic region. In the illustrated example, the increase reached a peak value of $36 \%$ above baseline level within a minute following the conjunction episode, and decayed thereafter to an apparent stable level of $15 \%$. The same test was performed on 10 slices (four animals) and showed an average increase of $12 \%(7-17)$ when measured 15 min after a conjunction consisting of five pairing events. This value of $12 \%$ can be compared with the average potentiation of $15 \%$ observed for an input subjected to homosynaptic tetanization with five impulses in picrotoxin solution (Wigström and Gustafsson, 1985b; see also Fig. 2C). Within the recording periods used here, up to $30 \mathrm{~min}$ following conjunction, the stability of the potentiation was similar to that of the potentiation induced by homosynaptic tetanization. This experiment thus shows that long-lasting potentiation can be induced when test responses given by single afferent volleys occur together with brief 
A

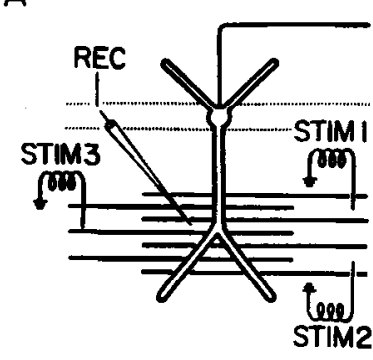

B

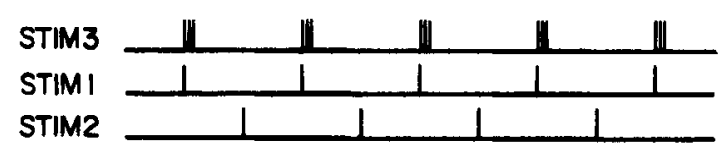

STIM3

STIMI

STIM2
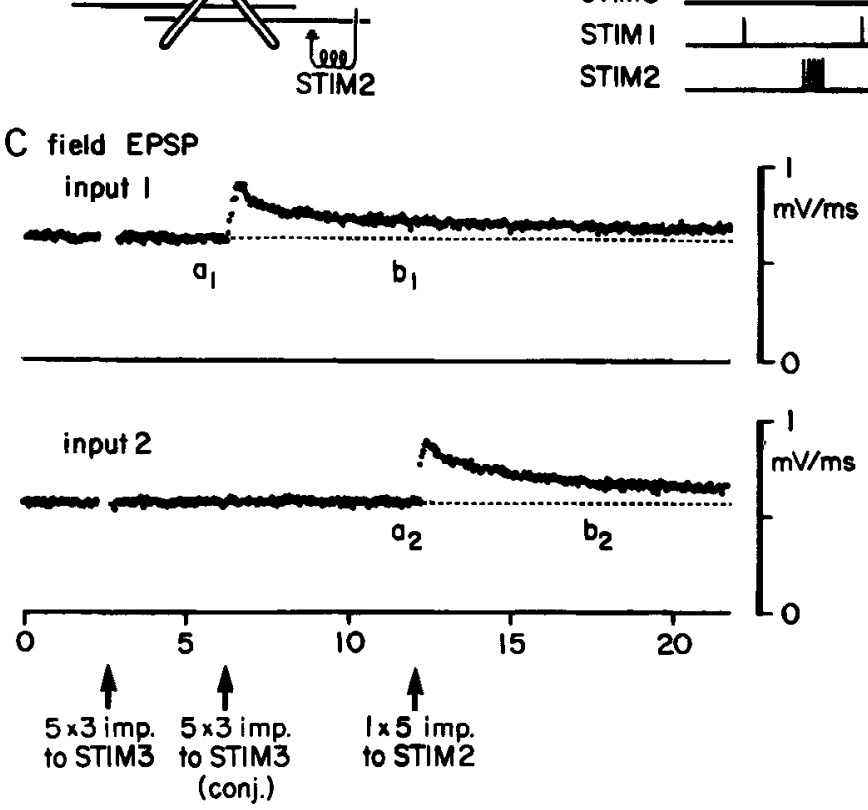

D

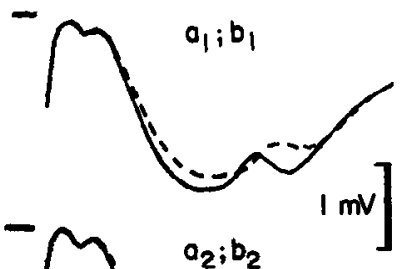

Figure 2. Comparison between LLP induced by conjunction and by homosynaptic tetanization. $A$, Diagram showing the arrangement of stimulating $(S T I M)$ and recording $(R E C)$ electrodes. $B$, Schematic drawing of the stimulus conditions during tetanization. Upper rows, Conjunction between test input $1(S T I M I)$ and the conditioning input 3 (STIM 3); lower rows, the single tetanus to input 2 (STIM 2). $C$. Measurements of the initial slopes of the field EPSPs resulting from activation of STIM 1 (input 1 ) and STIM 2 (input 2) are shown for a series of responses. $D$, Average records $(n=10)$ of the responses in $E$, taken at the indicated times.

tetani to a separate input, even when this conjunction occurs just a few times. As will be shown below, larger potentiation is obtained when using more conjunction episodes.

It can be noted in Figure $1 E$ (first and second arrows) that the test EPSP (input 2) was completely unaffected by tetanization of input 1 when there was a long delay $(2.5 \mathrm{sec})$ between the stimulations to the two inputs. This result should be expected from the previously shown input specificity of LLP (Andersen et al., 1977; McNaughton and Barnes, 1977). It also emphasizes the independence of the two inputs (see also Materials and Methods).

In other experiments, three stimulus electrodes were used, each giving rise to a separate input. In these experiments, the conditioning tetanus (three impulses) was positioned to occur $5 \mathrm{msec}$ after the test volley in order to allow for measurement of the test EPSP during the conditioning procedure. As shown in Figure $2 C$ (second arrow), pairing between the test input (input 1) and the conditioning tetanus (input 3, not plotted) resulted in a potentiation of the test input much as that described above in Figure $1 E$. There was, on the other hand, no change in test input 2 , which had not been paired with the conditioning tetanus ( $2.5 \mathrm{sec}$ delay). This experiment shows that potentiation occurs even when the test volley precedes the conditioning tetanus. It also shows that the LLP induced when the conditioning tetanus and the test input are combined is not caused by an unspecific effect due to the enhanced pre- and postsynaptic activity. If that were the case, one would also have expected test input 2 to increase. Test input 2 was subsequently tetanized once with five impulses at $50 \mathrm{~Hz}$ (Fig. $2 B$, lower part), i.e., with the same number of impulses as present for test input 1 during the five repeated pairing events. It can be observed in Figure $2 C$ that the potentiation of input 2 produced by homosynaptic tetanization much resembles that previously obtained for input 1 following conjunction, with much the same time course and magnitude (see above). In particular, both types of potentiation exhibit a similar initial transient phase of larger potentiation, suggesting that such a short-lasting potentiation phase is not necessarily a consequence of presynaptic postactivation changes (McNaughton, 1982).

In normal solution, i.e., in the absence of picrotoxin, brief trains containing 3-5 impulses are quite ineffective in producing potentiation of the tetanized input itself (Wigström and Gustafsson, 1985b); likewise, in normal solution (two animals), there was no change in a test input paired with such brief tetani (four slices). Using longer stimulus trains (20 impulses) repeated 5$10 \times$ generally leads to a large LLP in normal solution. However, even in these cases (five slices) there was practically no change of a test input when it was paired with the first volley of such trains.

\section{Time dependence of the conditioning effect}

It is clear from the above that the response to a single afferent volley can become potentiated if it occurs simultaneously with or even somewhat precedes a tetanus to a separate input. In order to establish more precisely the temporal requirements of this conjunction effect, an experiment similar to those described above was performed but with the test volley positioned at different times with respect to the conditioning train. For each set of inputs (generally for each slice), a single conditioning-test interval was normally used. In the cases where two different intervals were used, the interval initially used was such that the test input was minimally affected by its pairing with the conditioning train. The pairing event was repeated five times, and the amount of potentiation was measured $5 \mathrm{~min}$ later. To reduce the scatter of values a normalization procedure was used. Thus, after the test input had been conditioned and the resulting potentiation had reached a stable value, the test input itself (and in conjunction with the conditioning input) was tetanized twice with five impulses at $50 \mathrm{~Hz}$ and the degree of potentiation was measured 5 min after this "normalization" tetanus. The poten- 


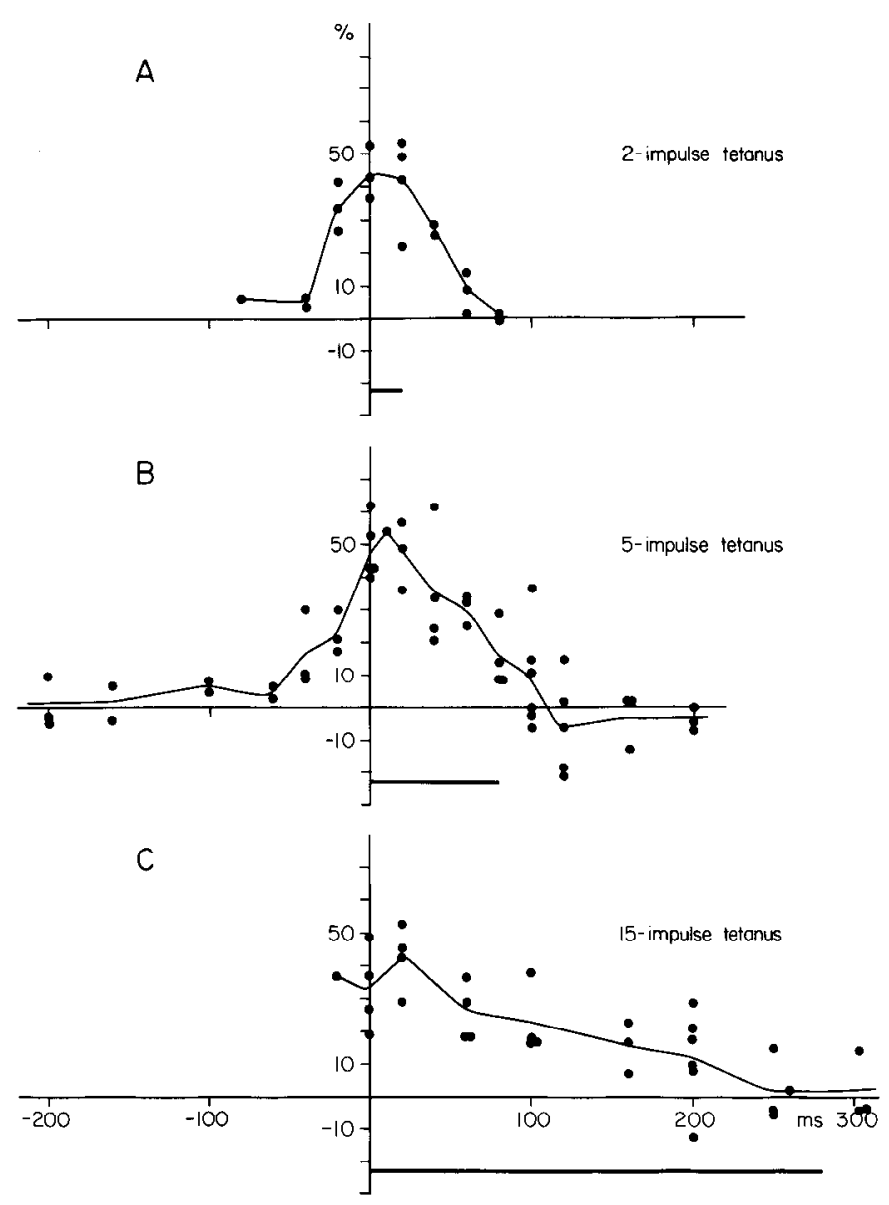

Figure 3. Time dependence of the effect of a conditioning tetanus. $A$, Effect of a 2-impulse tetanus. The origin of the time axis represents coincidence between the single test volley and the first volley in the train. Negative values (at left) on the same axis indicate that the test volley preceded the conditioning tetanus. The duration of the tetanus from the first to the last volley is shown below the graph as a thick line. In each slice, at most two (generally one) conditioning-test intervals were used, and the filled circles represent values obtained from each such trial. At each trial, the test EPSP was, after having reached a stable value (slope $=v 1$ ), combined with the conditioning train, and five such conditioning-test events were repeated at $0.2 \mathrm{~Hz}$. The slope of the test EPSP was measured $5 \mathrm{~min}$ thereafter (slope $=v 2$ ). The test and the conditioning inputs were subsequently twice tetanized together with a 5-impulse tetanus, and the test EPSP was measured after 5 min (slope $=v 3$ ). The degree of potentiation given by the conditioning tetanus was then calculated as $[(v 2-v 1) /(v 3-v 1)] \times 100$, i.e., as the fraction of the total potentiation that was obtained. The line in the graph is drawn through the average value at each interval. $B$ and $C$, Same as in $A$ but with a 5- and a 15 -impulse conditioning tetanus, respectively. The results were obtained from 10 animals.

tiation of the test input, given by the conditioning train only, was then expressed as a fraction of the total potentiation of the test input that had been obtained (see also legend to Fig. 3).

Figure $3 B$ shows the time dependence of the conditioning effect of a train of five impulses. Time $0 \mathrm{msec}$ in the graph indicates simultaneous activation of the test volley with the first volley in the train, and the bar below the graph indicates the duration of the train. The filled circles show the results from individual trials, and a line is drawn through the average value at each interval. It can be observed from the graph that the conditioning effect was largely restricted to the time of the tetanus. However, potentiation was also observed when the test volley preceded the train (to the left in the graph), the effect being substantial at a $20 \mathrm{msec}$ interval but decreasing thereafter to almost nil at intervals greater than $40 \mathrm{msec}$. The tetanus was not equally effective throughout its duration but had an optimal effect when the test occurred during the first $20 \mathrm{msec}$ of the train. The potentiating effect decreased thereafter, and was essentially over when the test volley occurred $20 \mathrm{msec}$ after the last volley in the train.

The same experiment was also performed with 2 and 15 volleys in the conditioning train. The short train of two impulses gave much the same peak potentiation as the 5 -impulse train and had only a somewhat briefer time course (Fig. $3 A$ ). Thus, in contrast to the longer train, the potentiating effect of this short train somewhat outlasted its own duration. The relative ineffectiveness of the later part of the 5-impulse tetanus was even more accentuated when using 15 volleys in the train (Fig. $3 \mathrm{C}$ ). In this case, the potentiation of the test input decreased continuously when moved later in time, and no potentiation was observed when the test was positioned during the last 3-4 volleys in the train.

\section{Relation between LLP induced by conditioning tetani and by homosynaptic tetanization}

In the foregoing sections it has been implicitly assumed that the potentiation observed when pairing single volleys with conditioning tetani is equivalent to that induced by tetanization of the test input itself. If this is so, one would not expect to observe any effect of a conditioning tetanus on an input that had previously been potentiated to a maximal level by homosynaptic tetanization, i.e., there would be an occlusion between the potentiations generated in these two manners. In these experiments, three independent inputs were used in much the same manner as previously shown in Figure 2 . One of the inputs (input 1) was first potentiated close to its saturation level by homosynaptic tetanization (Fig. $4 A$ ) and then combined with a conditioning tetanus (STIM 3, not shown). As illustrated in Figure $4 B$, the additional potentiation obtained during conjunction of this previously tetanized test input was quite small despite 40 pairing events, and essentially no potentiation remained 10 min after the end of the conditioning period. The same type of conjunction experiment was subsequently performed with the untetanized test input 2 . The conditioning was now effective (Fig. $4 C$ ), giving a potentiation of $30 \%$ when measured $10 \mathrm{~min}$ after the conjunction. This result suggests that a homosynaptically evoked LLP occludes that induced by conditioning tetani, as was observed in the four slices (two animals) examined in this manner.

As can be noted in Figure 4B, the effect of conjunction on the previously tetanized test input 1 was very transient, the EPSP increase declining already during the conjunction period. A similar decline also developed, however, in the nonpaired test input 2 during these more prolonged conditionings (not illustrated), suggesting some unspecific depressive action (note also a corresponding decline during conjunction in Fig. $4 C$ ). The relative decline of the paired and nonpaired test inputs was much the same, suggesting that in the absence of this depressive action, the increase of the paired test input would have stayed at the observed peak level throughout the conjunction period. On the other hand, the depression of the nonpaired test input disappeared within 1-2 min following the end of the conjunction period, implying that this unspecific depression was not responsible for the lack of long-lasting potentiation of the paired test input.

As illustrated in Figure $4, A$ and $C$, the potentiation induced in test input 2 by the conjunction is an appreciable fraction (0.6) of that observed for input 1 following homosynaptic tetanization. This raises the question as to what extent the capacity for potentiation can become saturated when inducing LLP by pairing single volleys and conditioning tetani. In order to examine this point, test inputs were combined with conditioning 


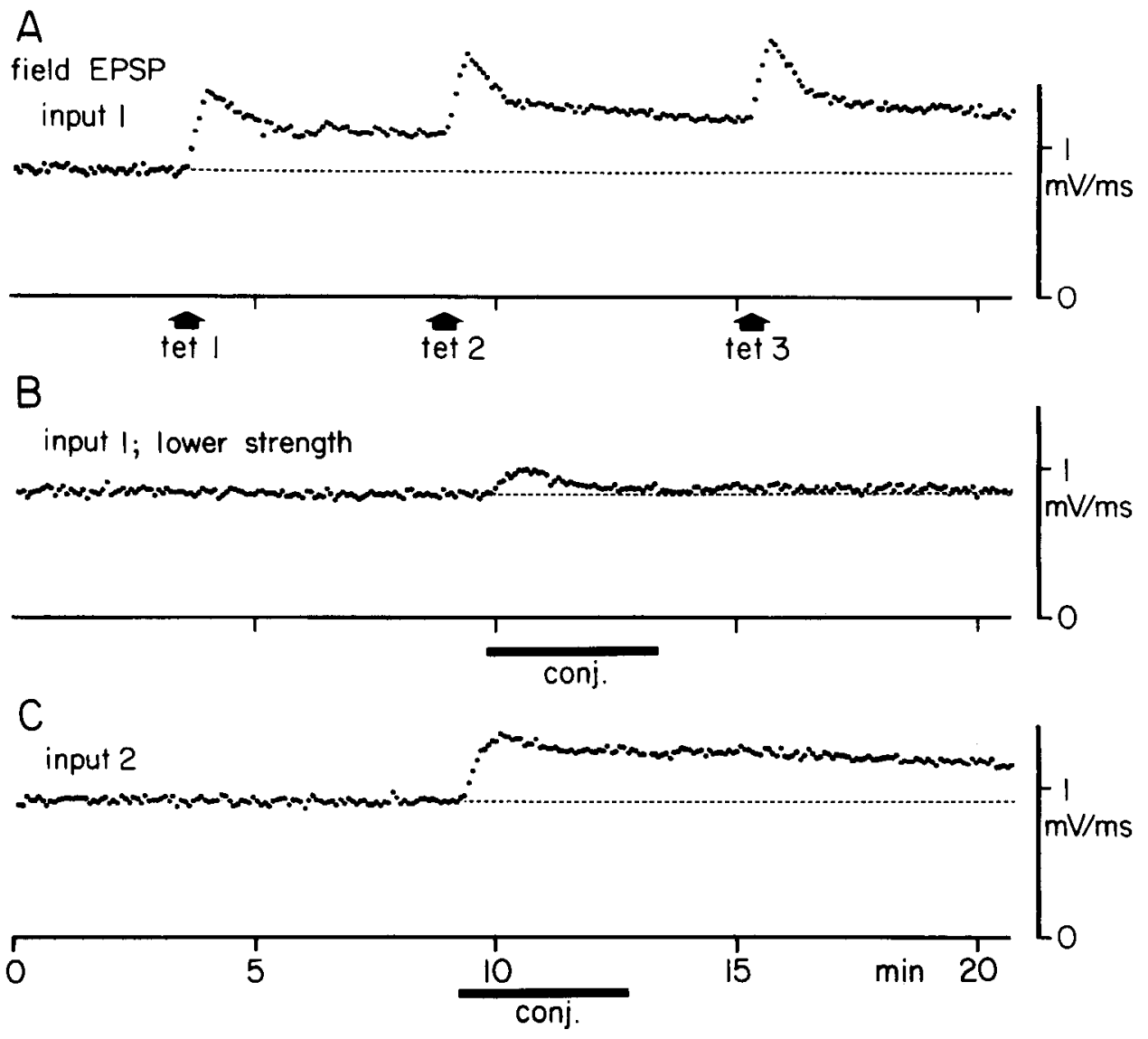

Figure 4. Occlusion between LLP induced by conjunction and by homosynaptic tetanization. $A-C$, Measurements of the initial slopes of field EPSPs plotted for a series of successive responses. The arrangement of stimulating and recording electrodes was as is illustrated in Figure 2. $A$ and $B$, Responses to STIM 1 (input 1); $C$, responses to STIM 2 (input 2). It should be noted that the measurements in $A$ $C$ are taken in a temporal sequence. The stimulation strength to STIM 1 is less in $B$ than in $A$ in order to reestablish the original test EPSP amplitude after the homosynaptic tetanization. The three tetanic episodes in $A$ each consisted of a three-impulse tetanus (at 50 $\mathrm{Hz}$ ) to input 1 repeated five times. $B$, Single volleys to input 1 were combined with three-impulse tetani to STIM 3, during the time interval indicated below the graph. $C$, Same procedure, but with input 2 as the test input. As discussed in the text, the decay of the potentiation appearing during the conditioning procedure corresponded well with a decrease in a test input not paired to the tetanus (not illustrated).

tetani (three impulses) and the pairing event was repeated 100 times (at $0.2 \mathrm{~Hz}$ ), i.e., far more than the 10-20 events needed to reach a peak level of potentiation (cf. Fig. 4C). The amount of potentiation observed $10 \mathrm{~min}$ following the conjunction averaged $29 \%(22-50, n=8$, three animals), which is almost half the $70 \%$ potentiation observed following homosynaptic tetanization with 100 impulses (Wigström and Gustafsson, 1985b). In six of the slices, the test input was subsequently homosynaptically tetanized with 100 impulses, which in all cases produced a further potentiation with respect to that induced by the conjunction. However, as could be anticipated from the occlusion noted above, the total amount of potentiation produced by conjunction plus homosynaptic tetanization $(67 \%$, on average) was no greater than that observed following homosynaptic tetanization alone (see above).

Following application of the $N$-methyl-D-aspartate (NMDA) receptor antagonist 2-amino-5-phosphonovalerate (APV), the LLP evoked by homosynaptic tetanization is blocked (Collingridge et al., 1983; Harris et al., 1984; Wigström and Gustafsson, 1984). In order to test the effect of APV on the conjunction effect, experiments were performed using three independent inputs (cf. Figs. 2, 4). Before APV application, one of the test inputs was paired $(20-30 \times)$ with the conditioning tetanus to ascertain the presence of the conjunction effect. APV ( $2 \mathrm{mM})$ was then applied as a drop to the surface of the slice (Wigström and Gustafsson, 1984), and the tetanus was paired the same number of times with the remaining test input. In the five slices (two animals) tested, no conjunction effect, even at short times, was observed following the APV application.

Spatial relation between test and conditioning inputs

In the experiments described above, the stimulating electrodes were all positioned in the stratum radiatum, i.e., in the apical dendritic layer. Thus, although the inputs were separate with respect to the activated afferent fibers, the terminals were likely located at neighboring regions of the dendritic tree. The question then arises whether a conditioning effect might also appear when pairing inputs to different regions of the dendritic tree. In Figure 5 is illustrated an example where the conditioning tetanus is evoked by a stimulating electrode in the middle of stratum oriens, i.e., in the basal dendritic layer, and the test volley by an electrode in the middle of the stratum radiatum (Fig. $5 \mathrm{~A}$ ). Repeated pairing of the test volley and the conditioning tetanus led to a potentiation of the test EPSP that lasted throughout the $20 \mathrm{~min}$ postconjunction observation period. The same result was observed in all eight slices examined (five animals), the amount of potentiation measured $10 \mathrm{~min}$ following conjunction varying from 5 to $29 \%$ with a mean value of $19 \%$. The effect of a conditioning input is thus not dependent on a close spatial relation to the test input, although a synaptic activation in the basal dendrites appears less effective than one projecting to the same dendritic region.

When pairing a test input in stratum radiatum with a brief train (five impulses, $50 \mathrm{~Hz}$ ) to the alveus (three animals), only small or no effects were observed. Even when using high stimulation strength $(30-50 \mu \mathrm{A})$ producing large population spikes in the cell body layer, no apparent potentiation appeared in $4 / 7$ slices, and in the remaining ones the potentiation was only about $5 \%$ when measured $10 \mathrm{~min}$ following conjunction. It thus appears that conditioning antidromic activation is much less effective than an orthodromic one. This result agrees with that of Lee (1983), who reported that LLP induced by an orthodromic tetanus is not enhanced if it is paired with an antidromic train. It is, however, clear that a more quantitative evaluation of the possible effect of antidromic tetani on the induction of LLP will require more direct control of the convergence of synaptic effects and antidromic activation onto the same postsynaptic neurons 


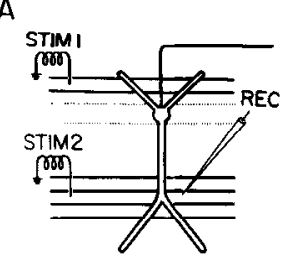

B

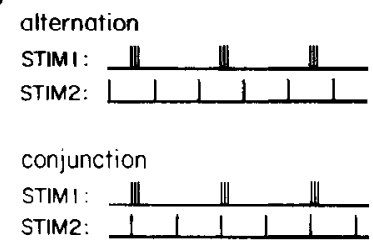

$\mathrm{C}$

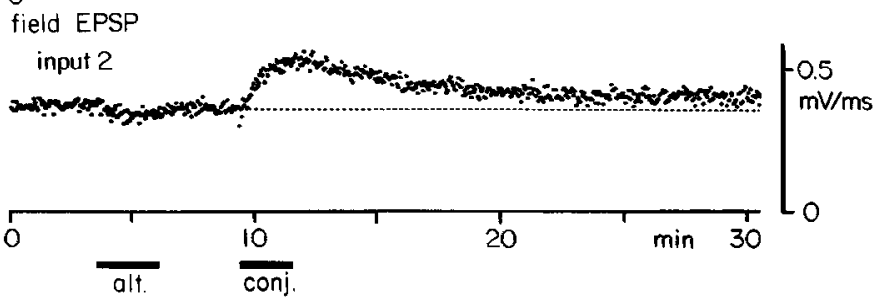

Figure 5. Conditioning effect of a stratum oriens input. $A$, Schematic diagram of the arrangement of stimulating $(S T I M)$ and recording $(R E C)$ electrodes. STIM 1 was used as a conditioning stimulus and the electrode was positioned in the stratum oriens. The test (STIM 2) and the recording electrode were located in the middle of stratum radiatum. $B$, Schematic drawing of the stimulus conditions during tetanization. During the conjunction tetanization, every second test volley coincided with the first volley of the conditioning tetanus, while in the alternation mode, the tetani occurred between the test volleys. $C$, Measurements of the initial slope of the field EPSP resulting from stimulation of input 2 are shown for a series of responses. The occurrence of two episodes of tetanization is indicated below the graph. During the alternation mode, there is a small decrease of the test response that quickly recovers after the end of the conditioning tetanization. During conjunction-type tetanization, there is a large increase of the test response that then decays to a stable elevated level.

as well as of possible current spread from the alveus stimulation site to afferents in stratum oriens.

\section{Discussion}

The present study shows that in picrotoxin-treated slices the response to a single afferent volley is potentiated when it occurs in conjunction with a conditioning tetanization to a separate set of afferents. Since the potentiation induced in this manner was long-lasting, was occluded by that evoked by tetanic activation of the test input itself, and was blocked by APV, it likely represents the same potentiation process as the LLP evoked by homosynaptic tetanization. The potentiation rose to a peak level with less than 20 conjunction events, and its value measured 10 min following conjunction was about half that which would have been evoked by homosynaptic tetanization itself. It thus appears that following reduction of postsynaptic inhibition longlasting potentiation of a test input can be effectively induced by simple conjunction with brief activity of other inputs. It should be noted that the proportion $(0.5)$ of total potentiation induced by conjunction alone would bc underestimated if the two inputs did not fully converge on the postsynaptic neurons recorded from. Even though the tests applied indicated a large degree of convergence (see Materials and Methods), it is likely that such a factor has contributed to the lower efficacy of conjunction relative to homosynaptic tetanization.

It was observed that the potentiation induced by conjunction did not only contain a long-lasting component but also a shortlasting component decaying within a few minutes. This conjunction-induced short-lasting potentiation was seen even after the long-lasting one had been saturated. Nevertheless, since the short-lasting component appeared only with the paired-test input and was blocked by APV, it may be a result of the same processes that lead to LLP.
For conjunction effects to occur, the test stimulus did not have to be exactly concurrent with the conditioning train as indicated by the time "windows" shown in Figure 3 for conditioning trains of different durations. These data are compatible with those of Levy and Steward (1983), who studied the cooperative interaction between trains of stimuli to separate inputs of the dentate gyrus in vivo. In that study, the LLP-producing effect was observed when the test train overlapped the conditioning train or preceded it by some $20 \mathrm{msec}$. Our results show that a very similar contiguity requirement also holds for the induction of LLP in the CA1 region of the slice preparation.

Cooperativity between afferents in producing LLP has previously been demonstrated in various regions of the hippocampus (Lee, 1983; Levy and Steward, 1979; McNaughton et al., 1978). Such effects have been observed also in the presence of GABA antagonists, suggesting that they can be unrelated to changes in inhibitory pathways (Barrionuevo and Brown, 1983; Wigström and Gustafsson, 1983c). These results suggest that the induction of LLP is controlled by a combination of pre- and postsynaptic activity, a notion also supported by the finding that the generation of LLP is greatly facilitated following reduction of inhibition (Wigström and Gustafsson, 1983a, b, 1985b). It would appear that the present results strongly support this suggestion, since LLP can be induced without any alteration of the presynaptic activity of the test input, the only change being the addition of a conditioning train evoking extra postsynaptic activity at a suitable time in relation to the test volley. The results also imply that the presynaptic requirement for LLP induction does not include any tetanic spike activity in the synaptic terminals or afferents.

It could be argued that the LLP-producing effect is not related to the postsynaptic activity elicited by the conditioning train, but to the fact that tetanic activation of conditioning afferents releases some substance, either directly or via activated interneurones, that diffuses to the terminals of the test input and there induces LLP. For this to be the case, such a substance must have its effect on activated synapses only, and its action would have to be fast as well as quickly terminated, since the conditioning effect of a 2-impulse train lasted less than 100 msec. Moreover, it must be as effectively released by a 2-impulse tetanus as by a 15-impulse one, and be inefficient at the end of a long train. It is difficult to imagine a presynaptic mechanism that meets all these requirements, particularly in view of the fact that an input to the basal dendritic region had a conditioning effect on a test input to the apical dendrites.

If it is then more likely that the conditioning effect is related to postsynaptic rather than presynaptic activity, such an effect could be mediated via changes in the extra- or intracellular environments, for example, in concentrations of potassium or calcium ions. However, while this type of mechanism cannot be excluded, it does not seem probable for the same reasons as outlined above. An alternative postsynaptic explanation of the LLP-producing effect of the conditioning train is its mediation via depolarization of the postsynaptic membrane. This alternative is of interest in view of a recently proposed mechanism for the induction of LLP (Wigström and Gustafsson, 1985a) implying that the process(es) leading to LLP may be initiated by an influx of calcium ions through voltage-dependent synaptic NMDA receptor channels located close to the non-NMDA receptor channels responsible for the EPSP normally observed. A single-volley EPSP contains a component that is blocked by APV and thus likely results from ionic flux through NMDA receptor channels (Wigström et al., 1985). According to the proposed idea, each single volley releases transmitter that interacts with both types of receptor, the current through the NMDA receptor channels being, however, normally small or nonexistent due to the rather small and short-lasting subsynaptic depolarization that is attained after a single volley. However, 
during tetanic activation, especially in the presence of GABA antagonists reducing inhibition, larger levels of subsynaptic depolarization are reached, the calcium influx through NMDA channels becomes exaggerated, and LLP is induced. With this kind of mechanism for LLP induction the subsynaptic depolarization needed to open the NMDA channels does not have to be provided by the specific input itself.

It may then be asked to what extent the observed timing curves, i.e., the relations between the conditioning-test intervals and the amount of LLP produced, agree with this interpretation of the mechanism underlying the conjunction effect. In order to indicate that the above mcchanism may account for the observed timing curves, a simplified representation is shown in Figure 6. It is assumed that the amount of LLP produced is directly related to the degree of overlap between two processes, one related to the transmitter-NMDA receptor interaction and the other to the subsynaptic depolarization given by a 2 -impulse conditioning tetanus. A timing curve resulting from the degree of overlap between two square waves used to approximate the two processes (see legend to Fig. 6) shows considerable similarities to that observed experimentally by pairing a single volley field EPSP with a 2-impulse tetanus (compare Fig. $6 C$ with Fig. $3 A$ ). LLP is in both cases produced when the test precedes the conditioning train (to the left in the graphs), the effect mainly disappearing with negative intervals of more than $40 \mathrm{msec}$. A more complete time course with this forward conditioning paradigm was obtained experimentally with 5 -impulse trains (Fig. $3 B$ ), revealing slight effects even up to $100 \mathrm{msec}$ intervals. This result is, however, well compatible with the discussed mechanism allowing for the fact that the $50 \mathrm{msec}$ duration only approximates the time to half-decay of the APV-blocked component.

The theoretically expected additional effect of a 5-impulse train on the timing curve is represented by the dashed line in Figure $6 \mathrm{C}$. It can be seen from experimental observations that the amount of LLP obtained with simultaneous activation of the test and the conditioning tetanus (time 0 ) was virtually independent of train duration-also produced by this type of mechanism. However, the predicted plateau level was not observed experimentally, especially with the 15 -impulse tetani with which virtually no potentiation was observed when the test was positioned during the late part of the tetanus. The existence of such a plateau rests, however, on the presumption that the subsynaptic depolarization stays at a high level throughout the tetanus. Even in the presence of picrotoxin and related GABA antagonists, there remains a later, more slowly developing, synaptic inhibition (Newberry and Nicoll, 1984), which will curtail the synaptic depolarization during the longer trains and thereby cause fewer conditioning effects. Moreover, the synaptically evoked firing of the cells will lead to spike-evoked afterhyperpolarizations (Hotson and Prince, 1980), which will further decrease the synaptic depolarization.

To summarize, the conjunction effects observed here may be explained by an interaction between one condition set by the test input, i.e., a synaptically mediated transmitter interaction with NMDA receptors, and another condition set by the conditioning tetanus, i.e., a postsynaptic depolarization achieved by activation of non-NMDA receptor channels. According to the present results, the appearance of these conjunction effects was dependent on the presence of picrotoxin in the bath. The present study was, for reasons outlined in the introduction, primarily concerned with an investigation of these phenomena in picrotoxin solution, and cannot exclude that, with more suitable timing between test volley and conditioning train and with additional pairings, conjunction effects would have appeared also in normal solution. In any event, with respect to the above interpretation of the data, the apparent absence of conjunction effects in normal solution is not surprising. In the normal so-
A transmitter - NMDA-receptor interoction

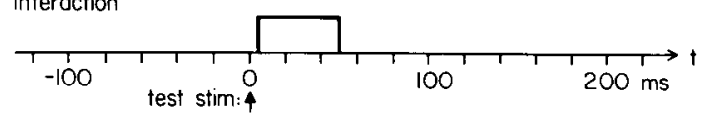

$B$ postsynaptic depolarization

(2 impulse train)

$\underset{-100}{r}, T_{1}$

C

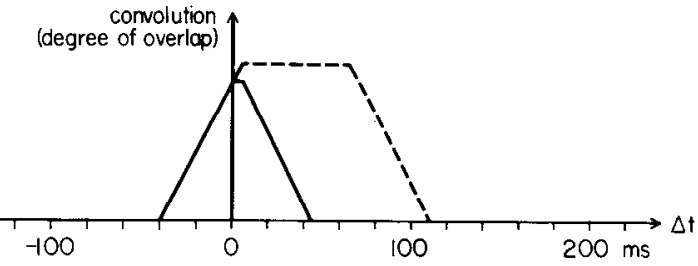

Figure 6. Schematic representation of a possible mechanism underlying the timing relations between test volley and conditioning train. $A$, Approximated time course of the interaction between transmitter and NMDA receptors following a single volley (evoked at time 0 , arrow) is indicated by square wave. As suggested by records such as that shown by Wigström et al. (1985, Fig. 2), the APV-blocked component appears to start some $5 \mathrm{msec}$ after stimulation onset and decays to half-amplitude in about $50 \mathrm{msec}$, and a square wave with this position in time has been used to represent the transmitter-NMDA receptor interaction. $B$, Approximated time course of the depolarization following a twoimpulse tetanus in picrotoxin (each impulse indicated by an arrow) is indicated by square wave with solid lines. This time course is based on intracellular recordings showing that the membrane potential is in the near-threshold region, or above, for about $10-50 \mathrm{msec}$ following the onset of a two-impulse tetanus. The additional depolarization given by a five-impulse train is shown with dashed lines. $C$, Curves indicate the change in the degree of overlap between the square waves in $A$ and $B$, when that in $A$ is moved in relation to that in $B$. Zero time refers to the position of the first volley in the train and negative time denotes that the test $(A)$ precedes the train $(B)$. Solid lines represent the interaction between a test volley and a two-impulse train, and the dashed lines indicate the additional effect given by a five-impulse train.

lution, the inhibition evoked during the conditioning tetanus as well as by the test volley itself will counteract the postsynaptic depolarization and its spread along the dendrites and thus the hctcrosynaptic opening of NMDA receptor channels belonging to a test input. The lack of observed conjunction effect in normal solution may then indicate that the present results are more relevant for the understanding of the LLP process than as a demonstration of a physiologically operating conditioning paradigm. However, interncurons involved in the inhibition are under control from various afferent pathways, and it seems not unreasonable to believe that under certain circumstances their activity can be such that the situation in an intact awake animal approaches that in picrotoxin-treated slices. In fact, it has been reported that in the intact awake animal, LLP can bc induced by trains containing as few as four stimuli (Douglas, 1977), a result closely resembling that observed in slices kept in picrotoxin than in normal solution.

\section{References}

Andersen, P., S. H. Sundberg, O. Sveen, and H. Wigström (1977) Specific long-lasting potentiation of synaptic transmission in hippocampal slices. Nature 266: 736-737.

Barrionuevo, G., and T. H. Brown (1983) Associative long-term potentiation in hippocampal slices. Proc. Natl. Acad. Sci. USA 80: 73477351.

Bliss, T. V. P., and A. C. Dolphin (1982) What is the mechanism of long-term potentiation in the hippocampus? Trends Neurosci. 5:289290. 
Bliss, T. V. P., and A. R. Gardner-Medwin (1973) Long-lasting potentiation of synaptic transmission in the dentate area of the unanaesthetized rabbit following stimulation of the perforant path. J. Physiol. (Lond.) 232: 357-374.

Bliss, T. V. P., and T. Lomo (1973) Long-lasting potentiation of synaptic transmission in the dentate area of the anaesthetized rabbit following stimulation of the perforant path. J. Physiol. (Lond.) 232: 331-356.

Collingridge, G. L., S. J. Kehl, and H. McLennan (1983) Excitatory amino acids in synaptic transmission in the schaffer collateral-commissural pathway of the rat hippocampus. J. Physiol. (Lond.) 334: 33-46.

Dolphin, A. C., M. L. Errington, and T. V. P. Bliss (1982) Long-term potentiation of the perforant path in vivo is associated with increased glutamate release. Nature 297: 496-498.

Douglas, R. M. (1977) Long-lasting synaptic potentiation in the rat dentate gyrus following brief high frequency stimulation. Brain Res. 126: 361-365.

Douglas, R. M., and G. V. Goddard (1975) Long-term potentiation of the perforant path-granule cell synapse in the rat hippocampus. Brain Res. 86: 205-215.

Douglas, R. M., G. V. Goddard, and M. Riives (1982) Inhibitory modulation of long-term potentiation: Evidence for a postsynaptic locus of control. Brain Res. 240: 259-272.

Eccles, J. C. (1983) Calcium in long-term potentiation as a model for memory. Neuroscience 10: 1071-1081.

Hablitz, J. J. (1984) Picrotoxin-induced epileptiform activity in hippocampus: Role of endogenous versus synaptic factors. J. Neurophysiol. 51: 1011-1027.

Harris, E. W., A. H. Ganong, and C. W. Cotman (1984) Long-term potentiation in the hippocampus involves activation of N-methyl-Daspartate receptors. Brain Res. 323: 132-137.

Hotson, J. R., and Prince, D. A. (1980) A calcium-activated hyperpolarization follows repetitive firing in hippocampal neurons. J. Neurophysiol. 43: 409-419.

Lee, K. S. (1983) Cooperativity among afferents for the induction of long-term potentiation in the CAl region of the hippocampus. J. Neurosci. 3: 1369-1372.

Levy, W. B., and O. Steward (1979) Synapses as associative memory elements in the hippocampal formation. Brain Res. 175: 233-245.

Levy, W. B., and O. Steward (1983) Temporal contiguity requirements for long-term associative potentiation/depression in the hippocampus. Neuroscience 8: 791-797.

Lømo, T. (1966) Frequency potentiation of excilatory synaptic activity in the dentate area of the hippocampal formation. Acta Physiol. Scand. 68(Suppl. 277): 128.

Lynch, G., and M. Baudry (1984) The biochemistry of memory: A new and specific hypothesis. Science 224: 1057-1063.
Lynch, G., J. Larson, S. Kelso, G. Barrionuevo, and F. Schottler (1983) Intracellular injections of EGTA block induction of hippocampal longterm potentiation. Nature 305: 719-721.

McNaughton, B. L. (1982) Long-term synaptic enhancement and shortterm potentiation in rat fascia dentata act through different mechanisms. J. Physiol. (Lond.) 324: 249-262.

McNaughton, B. L., and C. A. Barnes (1977) Physiological identification and analysis of dentate granule cell responses to stimulation of the medial and lateral perforant pathways in the rat. J. Comp. Neurol. 175: 439-454

McNaughton, B. L., R. M. Douglas, and G. V. Goddard (1978) Synaptic enhancement in fascia dentate: Cooperativity among coactive afferents. Brain Res. 157: 277-293.

Newberry, N. R., and R. A. Nicoll (1984) A bicuculline-resistant inhibitory post-synaptic potential in rat hippocampal pyramidal cells in vilro. J. Physiul. (Lond.) 348: 239-254.

Skrede, K. K., and D. Malthe-Sørenssen (1981) Increased resting and evoked release of transmitter following repetitive electrical tetanization in hippocampus: A biochemical correlate to long-lasting synaptic potentiation. Brain Res. 208: 436-441.

Skrede, K. K., and R. H. Westgaard (1971) The transverse hippocampal slice: A well-defined cortical structure maintained in vitro. Brain Res. 35: 589-593.

Wigström, H., and B. Gustafsson (1983a) Facilitated induction of hippocampal long-lasting potentiation during blockade of inhibition. Nature 301: 603-604

Wigström, H., and B. Gustafsson (1983b) Large long-lasting potentiation in the dentate gyrus in vitro during blockade of inhibition. Brain Res. 275: 153-158.

Wigström, H., and B. Gustafsson (1983c) Heterosynaptic modulation of homosynaptic long-lasting potentiation in the hippocampal slice. Acta Physiol. Scand. 119: 455-458.

Wigström, H., and B. Gustafsson (1984) A possible correlate of the postsynaptic condition for long-lasting potentiation in the guinea pig hippocampus in vitro. Neurosci. Lett. 44: 327-332.

Wigström, H., and B. Gustafsson (1985a) On long-lasting potentiation in the hippocampus: A proposed mechanism for its dependence on coincident pre- and postsynaptic activity. Acta Physiol. Scand. 123. 519-521.

Wigström, H., and B. Gustafsson (1985b) Facilitation of hippocampal long-lasting potentiation by GABA antagonists. Acta Physiol. Scand. 125: 159-172.

Wigström, H., B. Gustafsson, and Y.-Y. Huang (1985) A synaptic potential following single volleys in the hippocampal CAl region possibly involved in the induction of long-lasting potentiation. Acta Physiol. Scand. 124: 475-478. 